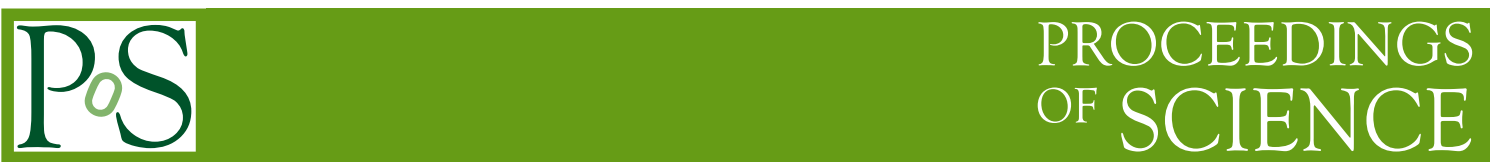

\title{
Diffuse axion-like particle searches
}

\author{
Ranjan Laha* \\ PRISMA Cluster of Excellence and Mainz Institute for Theoretical Physics, \\ Johannes Gutenberg-Universität Mainz, 55099 Mainz, Germany \\ E-mail: ranjalah@uni-mainz.de
}

\begin{abstract}
We discuss a new strategy to detect axion-like particles (ALPs) using the high-energy gammaray photons that are produced in or near sources of high-energy astrophysical neutrinos. These gamma-ray photons can convert to ALPs in the magnetic field of the source galaxy and can travel long distances. When these ALPs encounter the Milky Way magnetic field, they convert back to high-energy gamma-ray photons which can be detected by HAWC, CTA, and LHAASO. We show that this technique can probe ALP - photon coupling down to $10^{-11} \mathrm{GeV}^{-1}$ and ALP masses up to $3 \mu \mathrm{eV}$ in ten years of observation. This talk reports on Ref. [1]
\end{abstract}

Neutrino Oscillation Workshop (NOW2018)

9 - 16 September, 2018

Rosa Marina (Ostuni, Brindisi, Italy)

\footnotetext{
*Speaker.
} 


\section{Introduction}

The search for new physics is one of the main avenues of physics research. In this area, axionlike particles, characterized by their $g_{a \gamma} a \vec{E} \cdot \vec{B}$, where $g_{a \gamma}$ is the coupling strength, $a$ the ALP, $\vec{E}$ the photon's electric field, and $\vec{B}$ an external magnetic field, is a well motivated candidate. As a result of this coupling, photons can convert into ALPs (and vice versa) in the presence of magnetic fields. This process has been used to devise numerous techniques to search for ALPs [2]. In Ref. [1], we present a new technique to search for ALPs using this coupling.

The discovery of high-energy astrophysical neutrinos by the IceCube collaboration is one of the most important discoveries in astroparticle physics [3, 4]. Besides the coincidence with TXS 0506+056 [5], no other high-energy astrophysical neutrino sources have been identified and the high-energy astrophysical neutrino sky is isotropic. There has been numerous studies based on these neutrinos, with some focusing on better ways to distinguish signal to background [6], some on dark matter interpretations [7], some on the contributions to the atmospheric prompt neutrino flux [8], and some on new signal topologies in IceCube [9]. It is possible that astrophysical sources which produce these high-energy neutrinos are also producing high-energy gamma-rays via the $p-p$ and $p-\gamma$ interactions. In such a case, the neutrino and the photon might have comparable energies. These high-energy (energies between few $\mathrm{TeV}$ and a few $\mathrm{PeV}$ ) photons have a short mean free path (few Mpc to $\sim 10 \mathrm{kpc}$ ) compared to the extragalactic distances where these sources are located. As such, these photons are not expected to reach the Earth. The situation changes dramatically in the presence of ALPs. In addition to this high-energy photon flux, an additional contribution is produced by cosmic-ray interactions with the gas inside the Milky Way. We find that ALPs can leave a signature in this photon spectrum too.

\section{Search technique and results}

This search technique depends on a number of source parameters, which are as of now unknown. These are the magnetic field of the source, the redshift distribution of the sources, and the precise shape of the neutrino spectrum. We take the neutrino spectrum as

$$
\phi\left(E_{v}\right) \propto\left[\left(\frac{E_{v}}{E_{b}}\right)^{2}+\left(\frac{E_{v}}{E_{b}}\right)^{2 \alpha}\right]^{-\frac{1}{2}}
$$

where the neutrino energy is denoted by $E_{v}$, and $E_{b}=40$ (12) TeV is the break energy for the two cases that we consider, and the spectral index is $\alpha=2.92(2.48)$. The per-flavor normalization is $2.46(1.57) \times 10^{-18} \mathrm{GeV}^{-1} \mathrm{~cm}^{-2} \mathrm{~s}^{-1} \mathrm{sr}^{-1}$ at $E_{v}=100 \mathrm{TeV}$. We assume that the neutrinos are produced via the $p-\gamma$ interactions so that the energy of the photon, $E_{\gamma} \approx 2 E_{v}$. In order to tackle the uncertainty in the magnetic field, we assume that at $z=0$ the magnetic field strength follows the mean value given in Ref. [10]. We model the magnetic field via the domain model and assume that the domain length to be $1 \mathrm{kpc}$. We follow the discussion in Refs. [11, 12] to model the photon - ALP mixing. We assume that the magnitude of the extragalactic magnetic field is such that the photon - ALP mixing in that region is negligible.

When these ALPs reach the Milky Way, they can convert back into high-energy photons in the Galactic magnetic field. We model the Galactic magnetic field by following the Ref. [13]. ALP- 

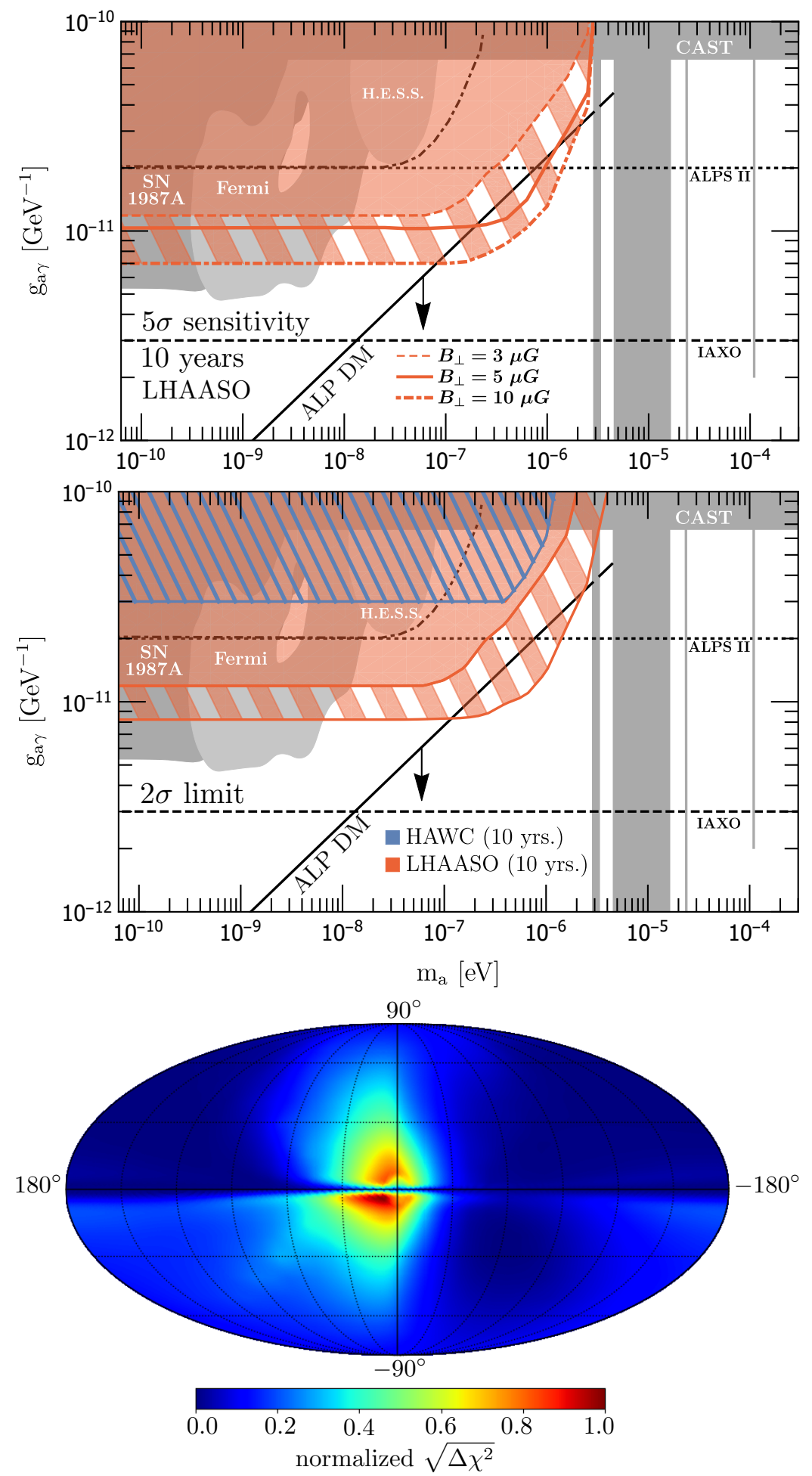

Figure 1: Top: LHAASO's sensitivity (red) to a diffuse ALP flux for the realistic scenario. The hatched region demonstrates the variation due to the source $\vec{B}$-fields $\left(B_{\perp}=3,5,10 \mu \mathrm{G}\right)$. We assume a coherence length of $1 \mathrm{kpc}$. The shaded regions are limits from CAST, various haloscopes, and astrophysics. We also show future sensitivities of CTA, ALPS-II, and IAXO. The solid black line ("ALP DM") shows the parameter space below which ALPs can act as dark matter. Middle: Limits from HAWC (blue) and LHAASO (red). Hatched regions show the uncertainty from our assumptions: lower (upper) boundary is for the realistic (conservative) scenario. The upper boundary is not visible for HAWC. See Ref. [1] for the relevant references. Bottom: Relative sensitivity to the photon-flux from a diffuse-ALP flux for a LHAASO-like experiment. 
photon conversion inside the Milky Way can be computed using

$$
i \frac{\mathrm{d}}{\mathrm{d} z} \rho=\left[\mathbf{H}_{\mathrm{dis}}, \rho\right]-\frac{i}{2}\left\{\mathbf{H}_{\mathrm{abs}}, \rho\right\}+i \mathbf{Q},
$$

where $\rho$ is the density matrix which contains particle densities and coherence, $\mathbf{H}_{\mathrm{dis}}$ and $\mathbf{H}_{\mathrm{abs}}$ are the dispersive and absorptive Hamiltonian respectively [12]. The source term for Galactic $p-p$ gamma-ray production is $\mathbf{Q}$.

Due to the isotropic distribution of the high-energy astrophysical neutrinos, the ALP flux that is incident on the Milky Way is also isotropic. However, due to the anisotropy of the regular Milky Way magnetic field, the resultant high-energy photons produced via this method have an anisotropic distribution on the sky (see right panel of Fig. 1). This morphology can be used to separate it from the background. The potential background is made up of high-energy gamma-ray produced via cosmic-ray interactions inside the Milky Way and is distributed along the plane.

The reach in the $g_{a \gamma}-m_{a}$ plane, where $m_{a}$ is the ALP mass, is shown in the left panel of Fig. 1 which is calculated assuming a ten year observation time window for HAWC and LHAASO. We see clearly that our technique can probe unconstrained parts of the parameter space. Although there are uncertainties at present, yet with more data the various inputs of our technique can be optimized. Due to the vastly different values of the photon mean free path (for the energies that we consider) and the source distance, it is very difficult for this signal to be confused with the background. This proposed signal is a smoking gun signature for ALPs and we hope that experimental data will show the true strength of this technique.

Acknowledgements: We thank all the organizers and participants of NOW 2018 workshop for the wonderful discussion. R.L. is supported by German Research Foundation (DFG) under Grant Nos. EXC-1098, KO 4820/1-1, FOR 2239, and from the European Research Council (ERC) under the European Union's Horizon 2020 research and innovation programme (grant agreement No. 637506, "vDirections") awarded to Joachim Kopp.

\section{References}

[1] H. Vogel, R. Laha and M. Meyer, arXiv:1712.01839 [hep-ph].

[2] I. G. Irastorza and J. Redondo, Prog. Part. Nucl. Phys. 102 (2018) 89 doi:10.1016/j.ppnp.2018.05.003 [arXiv: 1801.08127 [hep-ph]].

[3] M. G. Aartsen et al. [IceCube Collaboration], Phys. Rev. Lett. 111 (2013) 021103 doi:10.1103/PhysRevLett.111.021103 [arXiv:1304.5356 [astro-ph.HE]].

[4] M. G. Aartsen et al. [IceCube Collaboration], Astrophys. J. 833 (2016) no.1, 3 doi:10.3847/0004-637X/833/1/3 [arXiv:1607.08006 [astro-ph.HE]].

[5] M. G. Aartsen et al., Science 361 (2018) no.6398, doi:10.1126/science.aat1378 [arXiv:1807.08816 [astro-ph.HE]].

[6] R. Laha, J. F. Beacom, B. Dasgupta, S. Horiuchi and K. Murase, Phys. Rev. D 88 (2013) 043009 doi:10.1103/PhysRevD.88.043009 [arXiv:1306.2309 [astro-ph.HE]].

[7] K. Murase, R. Laha, S. Ando and M. Ahlers, Phys. Rev. Lett. 115 (2015) no.7, 071301 doi:10.1103/PhysRevLett.115.071301 [arXiv:1503.04663 [hep-ph]]. 
[8] R. Laha and S. J. Brodsky, Phys. Rev. D 96 (2017) no.12, 123002 doi:10.1103/PhysRevD.96.123002 [arXiv:1607.08240 [hep-ph]].

[9] M. D. Kistler and R. Laha, Phys. Rev. Lett. 120 (2018) no.24, 241105 doi:10.1103/PhysRevLett.120.241105 [arXiv:1605.08781 [astro-ph.HE]].

[10] A. Fletcher, ASP Conf. Ser. 438 (2011) 197 [arXiv:1104.2427 [astro-ph.CO]].

[11] A. Dobrynina, A. Kartavtsev and G. Raffelt, Phys. Rev. D 91 (2015) 083003 Erratum: [Phys. Rev. D 95 (2017) no.10, 109905] doi:10.1103/PhysRevD.91.083003, 10.1103/PhysRevD.95.109905 [arXiv:1412.4777 [astro-ph.HE]].

[12] A. Kartavtsev, G. Raffelt and H. Vogel, JCAP 1701 (2017) no.01, 024 doi:10.1088/1475-7516/2017/01/024 [arXiv:1611.04526 [astro-ph.HE]].

[13] R. Jansson and G. R. Farrar, Astrophys. J. 761 (2012) L11 doi:10.1088/2041-8205/761/1/L11 [arXiv:1210.7820 [astro-ph.GA]]. 